\title{
Internet-Based Cognitive Behavioral Therapy for Obsessive-Compulsive Disorder in Korea
}

\author{
Soon-Ho Seol ${ }^{1,2}$, Jun Soo Kwon ${ }^{1,3}$, Yang Yeol Kim², Sung Nyun $\mathrm{Kim}^{3}$, and Min-Sup Shin ${ }^{1,3 凶}$ \\ ${ }^{1}$ Department of Psychiatry, Seoul National University College of Medicine, Seoul, Republic of Korea \\ ${ }^{2}$ Seoultop Psychiatry Clinic \& Seoul Junior Counseling Center, Seoul, Republic of Korea \\ ${ }^{3}$ Department of Neuropsychiatry, Seoul National University Hospital, Seoul, Republic of Korea
}

\begin{abstract}
Objective Cognitive behavioral therapy (CBT) is regarded as one of the most effective intervention for obsessive-compulsive disorder $(\mathrm{OCD})$. However, many patients remain untreated or inadequately treated due to time or geographical constraints. The purpose of this study was to develop an internet-based CBT (ICBT) for OCD, and to examine its efficacy in the Korean clinical setting.

Methods The ICBT program ('COT') was developed from the same OCD manual in the standard CBT intervention. Twenty-seven participants of the total 42 patients completed all training sessions of the ICBT and the remainder $(\mathrm{n}=15)$ were classified as non-completers. Self-report measures of OCD, depression, anxiety, and work/social functioning, in addition to a neurocognitive test battery, were administered by face-to-face before and after treatment.

Results The participants showed significant improvements in OCD and depressive symptoms, and in work/social functioning after ICBT completion. The presence of combined medication had no significant impact on treatment effect. The non-completers displayed more severe depressive and anxiety symptoms, and ICBT responders were younger and performed better in the Wisconsin Card Sorting Test.

Conclusion ICBT was found to be as effective for patients with moderate OC symptoms and little treatment experience. Considering the high accessibility and convenience of ICBT, it could be a helpful first treatment step for OCD patients when face-to-face treatment is unavailable. In the future a randomized controlled study will be necessary for verification and generalization of these results.
\end{abstract}

Psychiatry Investig 2016;13(4):373-382

Key Words Obsessive-compulsive disorder, Cognitive behavioral therapy, Internet, Computer, Treatment efficacy.

\section{INTRODUCTION}

Obsessive-compulsive disorder (OCD) is characterized by the presence of obsessions and/or compulsions that are timeconsuming and cause clinically significant distress or functional impairment in social, occupational, or other important life areas. ${ }^{1}$ OCD can follow a chronic course if left untreated, ${ }^{2}$ and sufferers often experience impairment in multiple domains, resulting in reduced quality of life. ${ }^{3}$ Therefore, it is important that OCD patients have access to timely and effective treatment.

Received: April 27, 2015 Revised: October 30, 2015

Accepted: December 16, 2015 Available online: March 28, 2016

$\bowtie$ Correspondence: Min-Sup Shin, PhD

Department of Psychiatry, Seoul National University College of Medicine, 101 Daehak-ro, Jongno-gu, Seoul 03080, Republic of Korea

Tel: +82-2-2072-2454, Fax: +82-2-744-7241, E-mail: shinms@snu.ac.kr

(a) This is an Open Access article distributed under the terms of the Creative Commons Attribution Non-Commercial License (http://creativecommons.org/licenses/by$\mathrm{nc} / 3.0$ ) which permits unrestricted non-commercial use, distribution, and reproduction in any medium, provided the original work is properly cited.
Cognitive behavioral therapy (CBT) is known to be the most effective intervention for OCD, in conjunction with medication such as the selective serotonin re-uptake inhibitors (SSRI). ${ }^{4,5}$ Despite empirical evidence of the efficacy of this treatment approach, the majority of OCD patients are not in contact with a mental health professional, and less than $10 \%$ of patients actually received CBT in studies conducted in the UK and US. ${ }^{6,7}$

An approximately 10-year gap between OCD onset and treatment has been reported. ${ }^{8}$ Barriers to treatment-seeking include the direct and indirect costs of treatment, lack of time, distance to mental health services, and fear of stigma. ${ }^{9}$ Another possible reason is the limited number of CBT therapists working within the healthcare system..$^{10,11}$

This gap has precipitated increased focus on alternative delivery systems for OCD-based CBT, with the aim of providing the most effective treatment in a more accessible, less timeconsuming, and less costly manner. Accordingly, self-treatment using CBT manuals, ${ }^{12,13}$ interactive computer programs 
accessed via standalone PC or telephone (e.g., 'CAVE', 'BTSteps', etc.), ${ }^{14,15}$ and internet-based CBT (ICBT) (e.g., 'the OCD program, 'BiP OCD', etc.) ${ }^{16-18}$ have been developed over the past two decades. These self-administered treatments generally require less input from a therapist, and reduced patient costs, compared with face-to-face therapy. While there are several differences in treatment format (e.g., use of workbooks, interactive voice response software, internet, etc.), significant improvements in obsessive-compulsive (OC) symptoms were observed following short-term (8-15 sessions) application of these programs. In particular, medium to large effect sizes were observed for the primary outcome measure of the Yale-Brown Obsessive-Compulsive Scale (YBOCS): ${ }^{19}$ computer-guided behavioral therapy (BTSteps)= 0.8-1.2; ICBT $=1.53-2.29 .{ }^{15-18,20}$

However, standard (therapist-leaded) CBT showed higher effect size than self-treatment program (BTSteps), and patients who did not respond to BTSteps improved after switching to standard CBT. ${ }^{15}$ Because self-treatment is difficult to maintain, with higher dropout rates compared with face-to-face therapy, therapist support could be beneficial. ${ }^{20,21}$ Accordingly, it has been suggested that self-treatment programs are not 'clinician-replacers', but rather are 'clinician-extenders.' ${ }^{10}$

The UK National Institute of Clinical Excellence (NICE) ${ }^{5}$ suggests that a stepped-care approach be used for OCD, which subdivides treatment into brief, individual CBT with self-help material (step 3), multidisciplinary care in primary or secondary care (step 4), multidisciplinary care with expertise in OCD (step 5), and inpatient care or intensive treatment programs (step 6), depending on the complexity and difficulty of the case. Recently, a series of studies directly compared the effectiveness and direct/indirect cost of stepped care and standard exposure and response prevention (ERP) for OCD, and suggested that stepped care ERP can significantly reduce treatment costs without diminishing treatment efficacy and patient satisfaction. ${ }^{13,22}$

Self-treatment programs are expected to be useful and effective interventions for OCD patients in Korea, who are not receiving appropriate treatment due to prejudice and/or lack of mental health awareness. ${ }^{23}$ This study sought to utilize the new Korean ICBT program and to assess its efficacy in a clinical setting. We chose ICBT because of its easy accessibility and similar, or greater, effect sizes compared with other remote treatment protocols (e.g., bibliotherapy, BTSteps). We hypothesized that the Korean ICBT would result in significant improvements in OC symptoms, depression, anxiety, and general functioning.

Another aim of this study was to examine which participants are more responsive to ICBT. Little research on predictors of treatment response in ICBT has been thus far con- ducted. For this, factors that may affect compliance and the efficacy of ICBT, were also examined according to demographic, symptomatic, and neuropsychological domains. Especially, a qualitative review of clinical predictors of response to CBT for OCD reported that greater OC symptom severity predicts poorer treatment response. ${ }^{24}$ And many studies suggest executive function deficits in OCD patients, in for example planning, response inhibition, and set shifting/cognitive flexibility. ${ }^{25}$ Moreover, this executive dysfunction has been shown to relate to a poorer prognosis in OCD patients receiving $\mathrm{CBT}^{26}{ }^{2}$ So, we expected that the patients with milder OC symptom and better executive function would be more responsive to ICBT.

\section{METHODS}

\section{Participants and recruitment}

Ethical approval for this trial was granted by the Institutional Review Board at Seoul National University (C-0907012-285). Informed written consent was obtained from all study participants.

Participants were recruited from Seoul, Korea via referrals from mental health professionals or self-referral. Advertisements were placed in several universities, hospitals, mental health centers, and the websites of OCD self-help groups. Applicants who indicated an interest in the study were screened by a coordinator with a master's degree in Psychology, followed by a final face-to-face interview using the Structured Clinical Interview for DSM-IV Axis I Disorders, ${ }^{27}$ conducted by a licensed clinical psychologist. Participants who have not previously been diagnosed or treated for OCD were re-interviewed by a third psychiatrist to confirm their OCD diagnosis.

Inclusion criteria were as follows: aged 16-64 years, with a primary diagnosis of OCD according to the Diagnostic and Statistical Manual of Mental Disorders, 4th ed. criteria. ${ }^{1}$ Exclusion criteria were: 1 ) history of psychosis or bipolar disorder; 2) neurological disease (e.g., epilepsy, brain dysfunction, Tourette's disorder, etc.); 3) intellectual disability [estimated FIQ $<70$ in the Korean-Wechsler Adult Intelligence Scale (KWAIS) ]; 4) alcohol or drug abuse during the last 6 months; 5) minimal OC symptoms (total score $<14$ in the Korean selfreported Y-BOCS); 6) having previously undergone CBT for OCD; and 7) currently receiving any other psychological treatment. Combined use of medication was permitted, if the dose was stable for at least 2 weeks prior to the study's commencement, and if a participant's psychiatrist agreed to maintain a constant dosage throughout the study.

Between November 2009 and October 2012, a total of 109 respondents applied for the study, and 42 respondents were selected for participation through a final face-to-face inter- 
view. The sample size and reasons for exclusion are specified in a flowchart (Figure 1). Finally, twenty-seven participants completed all training sessions of the ICBT. The fifteen participant who dropped out were classified as non-completers and were excluded from post assessment and final analysis for treatment effect.

The demographic and clinical variables of the completers are presented in Table 1. Nine participants (33.3\%) were diagnosed with OCD for the first time during this study. Participants receiving combined medication were administered an average of 1.64 drugs; $54.5 \%(n=6)$ of these participants had a single SSRI prescription. In OCD subtype, the proportion of mixed-type OCD was high (33.3\%).

\section{Interventions and procedure}

The ICBT program was developed using the Korean CBT manual for $\mathrm{OCD},{ }^{28,29}$ which was based on pre-existing OCD CBT literature. ${ }^{30,31}$ The program comprised the following modules: 1) psycho-education pertaining to OCD and CBT; 2) anxiety-relieving techniques and ERP exercises; 3) identifying and restructuring dysfunctional beliefs related to OCD; and 4) relapse prevention.

Participants accessed the 'COT' program (www.ocdcbt. com) for a total of 11 sessions. It was recommended that they complete the course at a rate of one session per week. It took

Table 1. Demographic and clinical variables of the completers

\begin{tabular}{lc}
\hline \multicolumn{2}{c}{ Variable } \\
\hline Number of male (\%) & $19(70.4 \%)$ \\
Age & $29.22 \pm 9.03$ \\
Education (yr) & $14.96 \pm 2.28$ \\
Intelligence (K-WAIS FIQ) & $112.85 \pm 12.16$ \\
Handedness (Rt./ Lt./ Mixed) & $24 / 2 / 1$ \\
Onset age (yr) & $20.15 \pm 7.13$ \\
Duration of illness (yr) & $9.47 \pm 7.92$ \\
Duration of untreated period (yr) & $7.22 \pm 6.84$ \\
Number of patients on medication (\%) & $11(40.7 \%)$ \\
OCD subtype (checking/washing/obsession/ & $7 / 8 / 3 / 9$ \\
mixed) & \\
\hline
\end{tabular}

Data are presented as mean \pm SD. K-WAIS FIQ: Korean-Wechsler Adult Intelligence Scale, Full scale IQ, OCD: obsessive-compulsive disorder

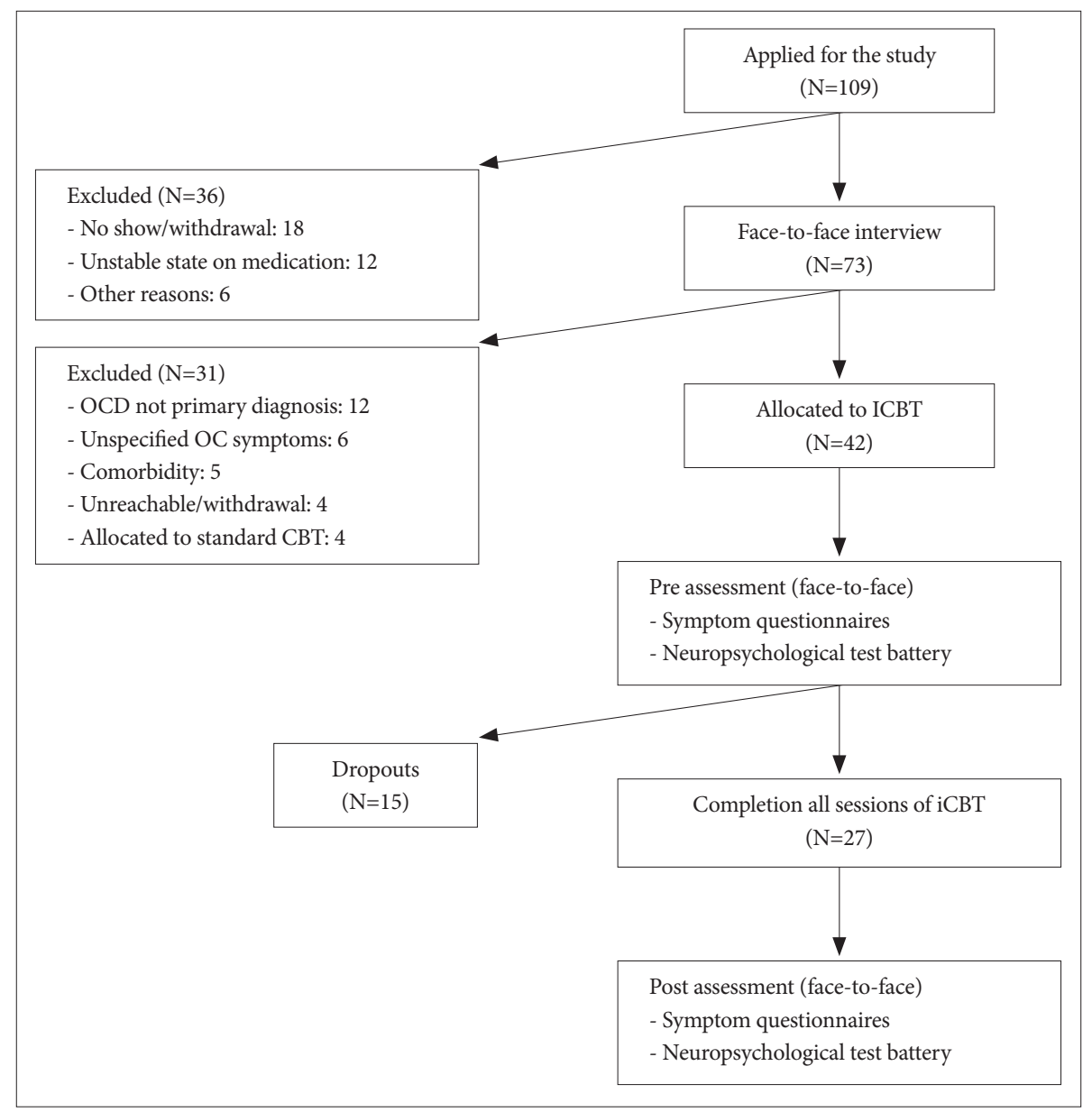

Figure 1. Participant flowchart. CBT: cognitive behavioral therapy, ICBT: Internet-based cognitive behavioral therapy. 
at least $5 \mathrm{~h}$ to complete certain sessions, including those featuring the ERP exercises, and approximately $1 \mathrm{~h}$ to complete the other sessions. It was possible to complete a session in stages, by accessing the site several times; however a limit of two consecutive sessions per day was imposed. Participants could raise questions at any time, via the Q\&A section of the website, or by emailing a coordinator. Answers were provided within 48 hours. In detail, a total of 87 question posts were registered in Q\&A section by thirty-one participant during research period (mean: 2.07; range 0-10). Major contents of questions were about program usage (e.g., program error correction, screen size adjustment, et al.), and specific inquiries for OCD treatment were limited. And a coordinator telephoned participants to identify any problems, and encourage treatment participation, if they had not logged on to the COT platform for 7 days or more. All participants received more than one call from a coordinator due to the login delays.

The COT program was designed to be available directly from the ERP training section of the website. In a pilot study, 24 typical triggers were identified for three OCD subtypes (i.e., checking, contamination, and pure obsession) (Figure 2). Each trigger took the form of photos, videos, and animations, and was divided into four exposure phases (vicarious or in-vivo exposure phase) for the purposes of systematic desensitization. Cognitive techniques such as cumulative odds ratio and pie chart could be automatically calculated on the website.

\section{Outcome measures}

Outcome measures were obtained pre- and post-treatment through the face-to-face meeting. The evaluators were graduate students in clinical psychology, or clinical psychology trainees at a university hospital, who were trained via two workshops.

The primary outcome measure of the study was the YBOCS, in line with previous studies. ${ }^{17,18}$ It comprises five obsession items and five compulsion items, rated on a 5-point Likert scale. In this study, the Korean self-report version of
Y-BOCS ${ }^{32}$ was used instead of the original clinician-administered Y-BOCS, because the self-report version has demonstrated strong convergent validity with the original version. ${ }^{15}$ Depressive symptoms were assessed by the Beck Depression Inventory (BDI), ${ }^{33}$ which is a 21 -item self-report scale that assesses the severity of various components of depression. The Beck Anxiety Inventory (BAI) ${ }^{34}$ contains 21 items that assess anxiety symptoms. All items are rated on a 5-point Likert scale in the BDI and the BAI. The Work and Social Adjustment Scale (WSAS) ${ }^{35}$ comprises five items of functional impairment, rated on a 9-point Likert scale, pertaining to work, home management, social leisure activities, private leisure activities, and the ability to form and maintain close relationships with others. At post-treatment, participants rated whether they had achieved their treatment goal, their COT training satisfaction, and the extent of their participation in the COT program according to a 100-point scale.

Finally, a neuropsychological test battery was conducted to investigate factors affecting treatment outcome. The battery comprised tests of memory and executive functioning; many previous studies of OCD have shown impairments in these domains, ${ }^{36}$ indexed by the following tasks: 1) Trail Making Test (TMT); ${ }^{37}$ 2) California Verbal Learning Test (CVLT); ${ }^{38} 3$ ) Rey-Osterrieth Complex Figure Test (ROCF); ${ }^{39}$ 4) Controlled Oral Word Association Test (COWA); ${ }^{40}$ 5) Stroop test; ${ }^{40} 6$ ) Object Alternation Test (OAT); ${ }^{41}$ and 7) the Wisconsin Card Sorting Test (WCST). ${ }^{42}$ The K-WAIS ${ }^{43}$ was also included to identify participants with sufficiently low intelligence scores to satisfy the exclusion criteria.

\section{Analysis}

All analyses were conducted using SPSS (ver. 20.0, IBM Co., Armonk, NY, USA). Paired-sample t-tests were used to examine improvements after treatment. Cohen's d formula, based on mean differences and pooled standard deviations, was used to calculate within-group effect sizes. A Cohen's d value of 0.5 or above was considered as a medium effect size,

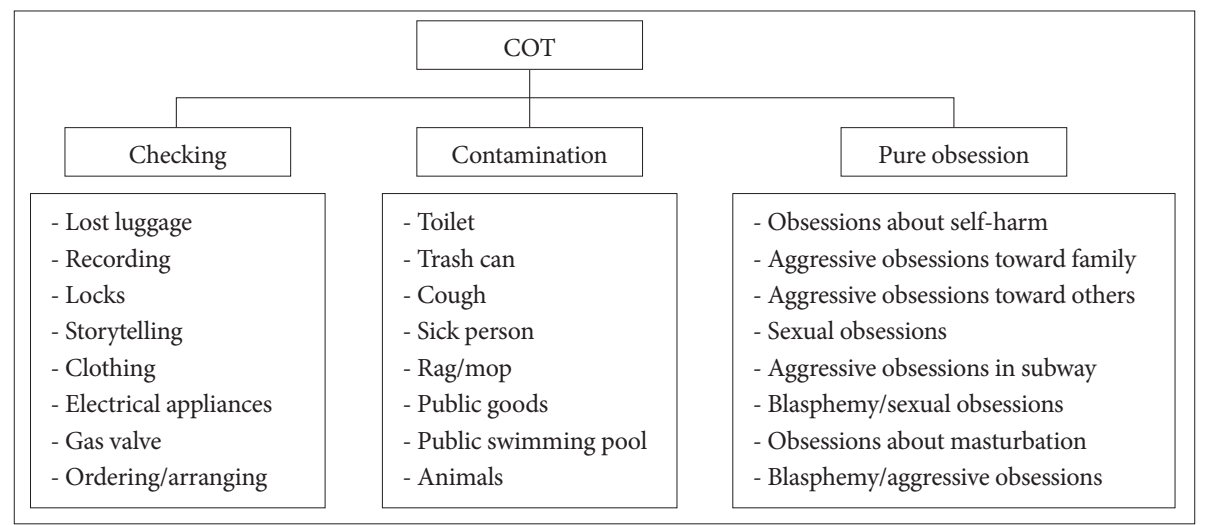

Figure 2. Configuration of COT triggers. COT: computerized obsessive-compulsive disorder therapy (Korean ICBT program). 
and 0.8 as a large effect size. We conducted additional analyses for several subgroups within ICBT as follows. Firstly, mixed model ANOVA (ICBT with medication vs. ICBT without medication) was used to identify the effects of combined medication use. Secondly, baseline differences were assessed between completers and non-completers in the participants., using independent t-tests for continuous variables and chisquare tests for categorical variables. Finally, clinically significant improvement was determined using the Jacobson \& Traux criteria ${ }^{44}$ in line with previous research: ${ }^{17} 1$ ) post-treatment scores of 14 or below; and 2) a 10-point or greater reduction on the Y-BOCS. We also employed the widely applied 35\% symptom severity reduction criterion on the YBOCS. ${ }^{45}$ Independent t-tests and logistic regression analysis were used to examine which demographic, symptomatic, and neuropsychological variables were significantly different between responders and non-responders, and therefore likely to be potential predictors of ICBT outcome.

\section{RESULTS}

\section{Treatment adherence and satisfaction}

Sixty-four percent (27/42) of participants completed all training sessions of the ICBT program. The major reasons for dropouts were reported as lack of time and difficulties maintaining their treatment motivation. Following completion of all training sessions, completers rated the treatment as relatively satisfied $(M=63.27, \mathrm{SD}=16.97)$ and reported that they participated in treatment relatively hard $(\mathrm{M}=67.88, \mathrm{SD}=17.16)$.

\section{Treatment efficacy}

The means and standard deviations of symptom questionnaires, for pre- and post-treatment, and paired t test and Cohen's d values, are presented in Table 2. Participants demonstrated significant improvements in OC symptoms, depressive symptoms, and work/social functionality following treatment. And a large within-group effect size was found on the YBOCS, while medium effect sizes were found for the BDI and WSAS. Although participants showed improvement for their anxiety symptoms, Cohen's d values were not significant.

\section{Comparisons according to combined medication}

Because $40.7 \%$ of the participants received medication, the treatment efficacy of ICBT alone, compared with ICBT plus medication, was additionally assessed (Table 3). Group differences before treatment were not significant for any outcome measure, Y-BOCS: $\mathrm{t}(25)=-0.80, \mathrm{p}=0.434$; BDI: $\mathrm{t}(25)=$ $0.84, \mathrm{p}=0.407$; BAI: $\mathrm{t}(25)=1.21, \mathrm{p}=0.236$; and WSAS: $\mathrm{t}(25)=$

Table 2. Means, SDs, t-values, and effect sizes of symptom questionnaires

\begin{tabular}{lcccc}
\hline & Pre-treatment & Post-treatment & Paired t-test & Cohen’s d \\
\hline Y-BOCS & $23.70(5.74)$ & $15.15(4.87)$ & $7.65^{* * *}$ & $1.64^{\ddagger}$ \\
Obsession & $11.78(2.59)$ & $7.74(2.52)$ & $6.83^{* * *}$ & $1.61^{\ddagger}$ \\
Compulsion & $11.93(3.42)$ & $7.41(2.47)$ & $7.39^{* * *}$ & $1.54^{\ddagger}$ \\
BDI & $14.89(8.89)$ & $9.37(8.75)$ & $3.17^{* *}$ & $0.64^{\dagger}$ \\
BAI & $14.26(8.19)$ & $10.93(9.90)$ & $2.07^{*}$ & 0.37 \\
WSAS & $17.70(10.01)$ & $11.19(7.84)$ & $2.81^{*}$ & $0.74^{\dagger}$ \\
\hline
\end{tabular}

Data are presented as mean (SD). ${ }^{*} \mathrm{p}<0.05,{ }^{* *} \mathrm{p}<0.01,{ }^{* * *} \mathrm{p}<0.001,{ }^{\dagger}$ medium effect size, ${ }^{\ddagger}$ large effect size. Y-BOCS: Yale-Brown ObsessiveCompulsive Scale (0-40), BDI: Beck Depression Inventory (0-63), BAI: Beck Anxiety Inventory (0-63), WSAS: Work/Social Adjustment Scale $(0-40)$

Table 3. Means, SDs, and F-values of symptom questionnaires for iCBT delivered with/without medication

\begin{tabular}{|c|c|c|c|c|c|c|c|}
\hline & \multicolumn{2}{|c|}{ ICBT with medication $(\mathrm{N}=11)$} & \multicolumn{2}{|c|}{ ICBT without medication $(\mathrm{N}=16)$} & \multirow{2}{*}{ F (Med) } & \multirow{2}{*}{$\mathrm{F}(\mathrm{Tx})$} & \multirow{2}{*}{$\mathrm{F}(\operatorname{Med} \times \mathrm{Tx})$} \\
\hline & Pre-treatment & Post-treatment & Pre-treatment & Post-treatment & & & \\
\hline Y-BOCS & $22.64(4.32)$ & $14.64(5.66)$ & $24.44(6.58)$ & $15.50(4.41)$ & 0.57 & $53.65^{* * *}$ & 0.16 \\
\hline Obsession & $11.36(2.16)$ & $7.55(2.91)$ & $12.06(2.89)$ & $7.87(2.31)$ & 0.40 & $42.79^{* * *}$ & 0.09 \\
\hline Compulsion & $11.27(2.41)$ & $7.09(2.84)$ & $12.38(3.98)$ & $7.62(2.25)$ & 0.68 & $49.88^{* * *}$ & 0.20 \\
\hline BDI & $16.64(6.92)$ & $8.09(8.15)$ & $13.69(10.06)$ & $10.25(9.28)$ & 0.02 & $11.91^{* *}$ & 2.16 \\
\hline BAI & $16.55(9.33)$ & $11.18(9.23)$ & $12.69(7.20)$ & $10.75(10.64)$ & 0.45 & $4.96^{*}$ & 1.09 \\
\hline WSAS & $15.82(9.60)$ & $11.45(7.89)$ & $19.00(10.38)$ & $11.00(8.06)$ & 0.27 & $6.75^{*}$ & 0.58 \\
\hline
\end{tabular}

Data are presented as mean (SD). ${ }^{*} \mathrm{p}<0.05,{ }^{* *} \mathrm{p}<0.01,{ }^{* * *} \mathrm{p}<0.001$. ICBT: internet-based cognitive behavioral therapy, Y-BOCS: Yale-Brown Obsessive-Compulsive Scale (0-40), BDI: Beck Depression Inventory (0-63), BAI: Beck Anxiety Inventory (0-63), WSAS: Work/Social Adjustment Scale (0-40), F (Med): F (Medication), between-group variance, F (Tx): F (Treatment), within-group variance, $F(\mathrm{Med} \times \mathrm{Tx})$ : $\mathrm{F}$ (Medication $\times$ Treatment), interaction effect 
-0.81, $\mathrm{p}=0.428$. A mixed-model ANOVA (ICBT with medication vs. ICBT without medication) for symptom scores indicated only main effects of treatment across all symptom questionnaires. A main effect of group, and a medication $\times$ treatment interaction effect, were not significant.

\section{Characteristics of non-completers}

Fifteen (35.7\%) of the 42 participants failed to complete the intervention. We investigated demographic, symptomatic, and neuropsychological differences between completers and non-completers (Table 4): no significant group differences were observed for demographic and neuropsychological variables, but non-completers exhibited significantly higher BDI and BAI scores compared with completers. In addition, the proportion of females was higher in the non-completer category, although this did not reach statistical significance.

\section{Characteristics of responders}

Seven participants (25.9\%) responded to ICBT according to Jacobson \& Traux's criteria. No significant differences between responders and non-responders were observed for any demographic, symptomatic, or neuropsychological variable.

When the 35\% reduction in Y-BOCS symptom severity criterion was applied, 14 participants (51.9\%) appeared to respond to treatment. Responders were younger, and made less total and perseverative errors in the WCST compared with non-responders (Table 5). No significant differences in any symptom questionnaire score were observed for responders vs. non-responders. When logistic regression analysis was performed, to include these three variables, omnibus tests of model coefficients were significant, $\chi^{2}=11.18, p<0.05$. However, no variable reached significance in terms of explanatory power, age: $\mathrm{B}=0.14, \mathrm{p}=0.069$; WCST, total errors: $\mathrm{B}=-0.05$, $\mathrm{p}=0.661$; and WCST, perseverative errors: $\mathrm{B}=-0.07, \mathrm{p}=0.592$.

Table 4. Comparison of completers and non-completers

\begin{tabular}{|c|c|c|c|c|}
\hline & Completers $(\mathrm{N}=27)$ & Non-completers $(\mathrm{N}=15)$ & Statistics & $\mathrm{p}$ \\
\hline Number of male (\%) & $19(70.4 \%)$ & $6(40.0 \%)$ & $3.69^{*}$ & 0.055 \\
\hline Age & $29.22 \pm 9.03$ & $28.67 \pm 10.32$ & $0.18^{\dagger}$ & 0.857 \\
\hline Education (yr) & $14.96 \pm 2.28$ & $14.27 \pm 2.15$ & $0.97^{\dagger}$ & 0.339 \\
\hline Intelligence (K-WAIS FIQ) & $112.85 \pm 12.16$ & $111.87 \pm 13.74$ & $0.24^{\dagger}$ & 0.811 \\
\hline Onset age (yr) & $20.15 \pm 7.13$ & $19.60 \pm 7.41$ & $0.24^{\dagger}$ & 0.815 \\
\hline Duration of illness (yr) & $9.47 \pm 7.92$ & $9.15 \pm 9.07$ & $0.12^{\dagger}$ & 0.906 \\
\hline Duration of untreated period (yr) & $7.22 \pm 6.84$ & $8.12 \pm 9.25$ & $-0.36^{\dagger}$ & 0.720 \\
\hline Number of patients on medication (\%) & $11(40.7 \%)$ & $9(60.0 \%)$ & $1.43^{*}$ & 0.336 \\
\hline OCD subtype (checking/washing/obsession/mixed) & $7 / 8 / 3 / 9$ & $4 / 2 / 6 / 3$ & $5.43^{*}$ & 0.143 \\
\hline Y-BOCS & $23.70 \pm 5.74$ & $25.53 \pm 6.97$ & $-0.92^{\dagger}$ & 0.365 \\
\hline BDI & $14.89 \pm 8.89$ & $22.67 \pm 12.01$ & $-2.20^{\dagger}$ & 0.039 \\
\hline BAI & $14.26 \pm 8.19$ & $26.40 \pm 13.45$ & $-3.18^{\dagger}$ & 0.005 \\
\hline WSAS & $17.70 \pm 10.01$ & $20.53 \pm 12.48$ & $-0.80^{\dagger}$ & 0.427 \\
\hline
\end{tabular}

Data are presented as mean \pm SD. ${ }^{*} \chi^{2}$ test, tindependent t-test. K-WAIS FIQ: Korean-Wechsler Adult Intelligence Scale, Full scale IQ, OCD: obsessive-compulsive disorder, Y-BOCS: Yale-Brown Obsessive-Compulsive Scale (0-40), BDI: Beck Depression Inventory (0-63), BAI: Beck Anxiety Inventory (0-63), WSAS: Work/Social Adjustment Scale (0-40)

Table 5. Comparison of responders and non-responders

\begin{tabular}{lcccc}
\hline & Responders* $^{*}(\mathrm{~N}=14)$ & Non-responders $(\mathrm{N}=13)$ & $\mathrm{t}$-value & $\mathrm{p}$ \\
\hline Age & $24.79 \pm 5.44$ & $34.00 \pm 9.85$ & -3.04 & 0.005 \\
Y-BOCS & $24.79 \pm 6.76$ & $22.54 \pm 4.37$ & 1.02 & 0.319 \\
BDI & $16.71 \pm 9.43$ & $12.92 \pm 8.16$ & 1.11 & 0.276 \\
BAI & $15.07 \pm 8.65$ & $13.38 \pm 7.92$ & 0.53 & 0.603 \\
WSAS & $16.36 \pm 8.84$ & $19.15 \pm 11.31$ & -0.72 & 0.479 \\
WCST, total errors $^{\dagger}$ & $52.86 \pm 8.73$ & $46.31 \pm 4.96$ & 2.37 & 0.026 \\
WCST, perseverative errors $^{\dagger}$ & $53.62 \pm 10.68$ & $45.15 \pm 5.43$ & 2.55 & 0.020 \\
\hline
\end{tabular}

Data are presented as mean \pm SD. ${ }^{*}$ responders were determined using the $35 \%$ Y-BOCS symptom severity reduction criterion, ${ }^{\dagger}$ these variables were presented as T-scores with a mean of 50 and a standard deviation of 10. Y-BOCS: Yale-Brown Obsessive-Compulsive Scale (0-40), BDI: Beck Depression Inventory (0-63), BAI: Beck Anxiety Inventory (0-63), WSAS: Work/Social Adjustment Scale (0-40), WCST: Wisconsin Card Sorting Test 


\section{DISCUSSION}

This study aimed to evaluate the efficacy of Korea's first ICBT program for OCD ('COT') in a clinical setting. Compared to the USA and Europe, ${ }^{46}$ Korea is at an early stage in the development, validation, and dissemination of self-treatment programs for OCD. Compared with existing programs (BTSteps, the OCD program, etc.), COT is more convenient, because all factors related to treatment are integrated into the COT platform, including the treatment manual, symptom evaluation, homework assignments, and ERP exercises. In particular, COT helps patients become familiar with the ERP exercises, because it is essentially composed of ERP triggers in the form of photos, videos, and animations. In addition, COT adds cognitive techniques, which are expected to be effective when applied to patients with pure obsessions. ${ }^{12}$

\section{The treatment efficacy and advantages of ICBT}

After completion of ICBT program, significant improvement was observed on the primary and secondary outcome measures. Especially, total Y-BOCS scores, of 23 points or above, fell to 15 points following treatment, below the 16-point diagnostic cutoff point. ${ }^{47}$ The within-group effect size (1.64) in this study was quite high compared with the effect size $(0.8$ in BTstep) reported by Greist et al. ${ }^{15}$ Our results are similar to the effect size (1.53-1.55) reported for other ICBT programs. ${ }^{17,18}$ Andersson et al. ${ }^{18}$ concluded that this owes to the high-intensity of the intervention and feedback provided by therapists within the ICBT program. However, the present study maintained the therapist's involvement at a minimum level. A greater therapeutic effect compared with BTSteps may be attributable to the easy accessibility of information, and inclusion of direct ERP exercises, in our internet-based platform, compared with the interactive voice response system of BTSteps. While other studies ${ }^{15,18}$ included the data for noncompleters in statistical analysis, this study excluded them from final analysis for pre-post treatment effect, which can inflate the within-group effect size of ICBT.

While depressive symptoms and work/social functional impairment diminished significantly, only anxiety symptoms failed to improve significantly. Because the BAI is more sensitive to panic disorder than OCD, ${ }^{48}$ improvement in OC symptoms cannot be accurately appraised by the BAI. Otherwise, the physiological anxiety levels of OCD patients may remain largely unchanged following $\mathrm{CBT}^{49}$ When separating the four subscales of the Korean version of the BAI, ${ }^{50}$ and comparing them before and after treatment, only subjective symptom scores were significantly reduced, $\mathrm{t}(25)=2.30, \mathrm{p}<0.05$. Physiological anxiety scores (neurophysiological, autonomic, and panic symptoms) were not significantly different after treatment.

ICBT has proven to be effective for OCD patients with moderate OC symptoms and little treatment experience from this results. The presence of combined medication had no significant impact on treatment effect. In addition, ICBT requires less time and effort from therapists compared with face-to-face CBT. In this study, ICBT needed a total of 4 hours from therapist, including the screening interview and psychological assessment. There were no face-to-face meetings during the implementation of the COT program and all contacts were made by telephone or via the Internet, which can be primarily managed by the coordinators.

ICBT can serve as a powerful therapeutic alternative for patients who forego traditional CBT due to time, monetary constraints, and/or stigma. Many participants (workers, students, and local residents) in this study actually preferred to be involved in ICBT than in face-to-fact treatment. Scores for COT training satisfaction and COT program completion were high (60/100 and above). Other benefits of ICBT include the fact that clinician training is not required, and treatment elements can be added or removed as appropriate. ${ }^{15}$

\section{The limitations of ICBT}

Not all participants achieved good results from ICBT in the present study. The dropout rate of participants was $35.7 \%$, which was higher compared with ones (12-14\%) in previous studies. ${ }^{18,20}$ This is because they defined non-completers as participants who did not undertake the ERP exercise to be corresponded to 'Module $5^{18}$ or 'Step $4,{ }^{20}$ while our study defined non-completers as participants who did not complete all training sessions. Applying this non-completer criterion to our study, 9 (21.4\%) of 42 participants dropped out before starting the ERP exercise module (i.e., the 4th session), which represents a similar dropout rate to the previous studies. However, considering that the intervention of the therapist in this study was kept to minimum level compared to other studies, dropout rate would have been reduced if the regular therapist contact was available.

Non-completers exhibited higher levels of depression and anxiety compared with completers at pre-treatment, which can negatively impact motivation for self-treatment and/or render it more difficult to endure triggers in the ERP exercises. The high proportion of female non-completers may relate to their slightly higher levels of depression and anxiety compared with the males, BDI: $\mathrm{t}(25)=-1.98, \mathrm{p}=0.059$; and BAI: $\mathrm{t}$ $(25)=-2.04, p=0.051$. For completers, completion of the entire COT program took an average of 18 weeks (mean: 127 days; range: $65-240$ days), considerably more time than the 10 weeks originally planned. It may be useful to adjust for the speed of individual participants; in the study by Greist et 
al., ${ }^{15}$ participants were permitted to proceed in BTSteps at their own pace.

Motivation and compliance are particularly important for self-treatment. It is firstly necessary to foster participant motivation through regular contact with coordinators and therapists, as noted in several studies. ${ }^{18,20}$ An important research question for the future will be how much therapist support is required to achieve optimal cost-effectiveness without impairing efficacy. ${ }^{51}$ In addition, efforts are required to identify and apply other treatment-enhancing factors in the future. For example, use of audio-visual materials, such as videos and animations, are helpful, as is increasing the opportunity for interaction as opposed to passively reading the treatment manual. Possible further development of ICBT may involve the combination of web-based ICBT with integrated smartphone application. ${ }^{51}$ Smartphone application could prompt the participants in ERP between sessions, thus potentially increasing adherence and overall outcome..$^{52}$ As suggested in the stepped care model of OCD,${ }^{10}$ it may be more useful to apply ICBT as a primary treatment for patients with greater insight into, and motivation for, treatment. Participants' levels of depression and anxiety should also be considered.

\section{The predictors of response to ICBT}

Another aim of this study was to examine which participants are more responsive to ICBT. In the present study, ICBT responders were younger and exhibited superior executive functioning measured by the WCST, compared with non-responders. A recent meta-analysis of CBT outcome for OCD reported a significant relationship between effect size and mean age; older age was associated with smaller effect sizes. ${ }^{53}$ Younger age may actually be associated with greater responsiveness to $\mathrm{CBT}^{54}$ but an alternative interpretation is that young people may be more adept at accessing and utilizing an internet-based program such as ICBT. However, when comparing ICBT efficacy for depression and for other anxiety disorders, the programs are reported to be effective across the entire age spectrum, including even those aged 60 years and above. ${ }^{55}$

As a possible predictor of non-response, the rationale for targeting neurocognitive impairment is predicated on the assumption that deficits in abstract-logical thinking may limit a patient's ability to comprehend issues discussed during psychological interventions, and also that deficits in mental flexibility may decrease patients' ability to transfer skills acquired in therapy to everyday life. ${ }^{56}$ However, it is difficult to draw conclusions regarding neuropsychological predictors of CBT outcome, because inconsistent results have been reported across different samples and neuropsychological tests. ${ }^{57}$ In the present study, executive functioning measured by the WCST did not possess significant explanatory power within the logistic regression analysis: further studies with larger samples of $\mathrm{OCD}$ patients are thus required.

Finally, our prediction that the patients with milder OC symptom would be more responsive to ICBT was not supported. The prior meta-analysis ${ }^{53}$ of CBT outcome reported that higher pretreatment OC symptom severity was not significantly associated with lower CBT effect size. However, researchers suggested that it may be that only severe OCD hinders outcome, and difference of treatment reactivity can be observed by separating the treatment groups depending on the baseline OCD severity.

The limitations of this study were as follows: a randomized controlled design was not used owing to recruitment difficulties. In the future a randomized controlled study will be necessary for verification and generalization of these results. Secondly, the long-term therapeutic effects of ICBT were not determined in this study. There is a need to check symptom change at 6 and 12 months following completion of the COT program. Thirdly, the small sample size did not allow for assessment of therapeutic efficacy according to OCD subtype. If OCD subtypes are identified using structured questionnaires, and their response to treatment assessed separately, more extensive discussion regarding the efficacy of ICBT would be possible.

This study was the first to assess the therapeutic efficacy of the Korean ICBT program for OCD in a clinical setting. Despite several limitations, the results suggests that COT program can rapidly and effectively aid OCD patients with moderate OC symptoms and little previous treatment experience. For patients already receiving therapist-lead CBT, ICBT can act as an adjunct, by providing homework assignments between sessions, or relapse prevention techniques following completion of face-to-face treatment. ICBT could also be useful for individuals with subclinical levels of OC symptoms, who do not need to attend a hospital.

\section{Acknowledgments}

This research was supported by National Research Foundation grants funded by the Ministry of Education, Science and Technology in Korea (No: 800-20090145). Min-Sup Shin, Jun Soo Kwon, and Soon-Ho Seol hold the patent for ICBT program for OCD in Korea (Patent No. 10-1135250).

\section{REFERENCES}

1. Association Psychiatric Association. Diagnostic and Statistical Manual of Mental Disorders, 4th Edition. Washington: American Psychiatric Association; 1997.

2. Mataix-Cols D, Rauch SL, Baer L, Eisen JL, Shera DM, Goodman WK, et al. Symptom stability in adult obsessive-compulsive disorder: data from a naturalistic two-year follow-up study. Am J Psychiatry 2002; 159:263-268.

3. Olatunji BO, Cisler JM, Tolin DF. Quality of life in the anxiety disorders: a meta-analytic review. Clin Psychol Rev 2007;27:572-581.

4. Foa EB. Cognitive behavioral therapy of obsessive-compulsive disor- 
der. Dialogues Clin Neurosci 2010;12:199-207.

5. National Institute for Clinical Excellence. Obsessive-Compulsive Disorder: Core Interventions in the Treatment of Obsessive-Compulsive Disorder and Body Dysmorphic Disorder. London: National Institute for Clinical Excellence; 2005.

6. Blanco C, Olfson M, Stein DJ, Simpson HB, Gameroff MJ, Narrow WH. Treatment of obsessive-compulsive disorder by U.S. psychiatrists. J Clin Psychiatry 2006;67:946-951.

7. Torres AR, Prince MJ, Bebbington PE, Bhugra DK, Brugha TS, Farrell $\mathrm{M}$, et al. Treatment seeking by individuals with obsessive-compulsive disorder from the british psychiatric morbidity survey of 2000. Psychiatr Serv 2007;58:977-982.

8. Marques L, LeBlanc NJ, Weingarden HM, Timpano KR, Jenike M, Wilhelm S. Barriers to treatment and service utilization in an internet sample of individuals with obsessive-compulsive symptoms. Depress Anxiety 2010;27:470-475.

9. Collins KA, Westra HA, Dozois DJ, Burns DD. Gaps in accessing treatment for anxiety and depression: challenges for the delivery of care. Clin Psychol Rev 2004;24:583-616.

10. Mataix-Cols D, Marks IM. Self-help with minimal therapist contact for obsessive-compulsive disorder: a review. Eur Psychiatry 2006;21:75-80.

11. Yang JC, Ha TH, Kim W, Kim SJ, Koo MS, Kwon JS, et al. Korean treatment algorithm for obsessive-compulsive disorder 2007 (III): a preliminary study for application of cognitive-behavioral therapy. Korean J Psychopharmacol 2007;18:408-413.

12. Moritz S, Jelinek L, Hauschildt M, Naber D. How to treat the untreated: effectiveness of a self-help metacognitive training program (myMCT) for obsessive-compulsive disorder. Dialogues Clin Neurosci 2010;12:209-220.

13. Tolin DF, Diefenbach GJ, Gilliam CM. Stepped care versus standard cognitive-behavioral therapy for obsessive-compulsive disorder: a preliminary study of efficacy and costs. Depress Anxiety 2011;28:314-323.

14. Clark A, Kirkby KC, Daniels BA, Marks IM. A pilot study of computer-aided vicarious exposure for obsessive-compulsive disorder. Aust N Z J Psychiatry 1998;32:268-275.

15. Greist JH, Marks IM, Baer L, Kobak KA, Wenzel KW, Hirsch MJ, et al. Behavior therapy for obsessive-compulsive disorder guided by a computer or by a clinician compared with relaxation as a control. J Clin Psychiatry 2002;63:138-145.

16. Lenhard F, Vigerland S, Andersson E, Ruck C, Mataix-Cols D, Thulin U, et al. Internet-delivered cognitive behavior therapy for adolescents with obsessive-compulsive disorder: an open trial. PLoS One 2014;9:e100773.

17. Wootton BM, Titov N, Dear BF, Spence J, Andrews G, Johnston L, et al. An internet administered treatment program for obsessive-compulsive disorder: a feasibility study. J Anxiety Disord 2011;25:1102-1107.

18. Andersson E, Enander J, Andren P, Hedman E, Ljotsson B, Hursti T, et al. Internet-based cognitive behaviour therapy for obsessive-compulsive disorder: a randomized controlled trial. Psychol Med 2012;42:21932203.

19. Goodman WK, Price LH, Rasmussen SA, Mazure C, Fleischmann RL, Hill CL, et al. The Yale-Brown obsessive compulsive scale: I. Development, use, and reliability. Arch Gen Psychiatry 1989;46:1006-1011.

20. Kenwright M, Marks I, Graham C, Franses A, Mataix-Cols D. Brief scheduled phone support from a clinician to enhance computer-aided self-help for obsessive-compulsive disorder: randomized controlled trial. J Clin Psychol 2005;61:1499-1508.

21. Andersson E, Ljotsson B, Hedman E, Kaldo V, Paxling B, Andersson $\mathrm{G}$, et al. Internet-based cognitive behavior therapy for obsessive compulsive disorder: a pilot study. BMC Psychiatry 2011;11:125.

22. Gilliam CM, Diefenbach GJ, Whiting SE, Tolin DF. Stepped care for obsessive-compulsive disorder: an open trial. Behav Res Ther 2010;48: 1144-1149.

23. Cho MJ. The 2011 Epidemiological Survey of Mental Disorders among Korean Adults. Seoul: Ministry of Health and Welfare; 2011.

24. Keeley ML, Stroch EA, Merlo LJ, Geffken GR. Clinical predictors of response to cognitive-behavioral therapy for obsessive-compulsive disorder. Clin Psychol Rev 2008;28:118-130.

25. Abramovitch A, Abramowitz JS, Mittelman A. The neuropsychology of adult obsessive-compulsive disorder: a meta-analysis. Clin Psychol Rev 2013;33:1163-1171.

26. Cavedini P, Riboldi G, D’Annucci A, Belotti P, Cisima M, Bellodi L. Decision-making heterogeneity in obsessive-compulsive disorder: ventromedial prefrontal cortex function predicts different treatment outcomes. Neuropsychologia 2002;40:205-211.

27. Spitzer RL, Gibbon M, Williams JB. User's Guide for the Structured Clinical Interview for DSM-IV Axis I Disorders [SCID-I]: Clinician Version. Washington, DC: American Psychiatric Press; 1997.

28. Shin MS, Seol SH. Cognitive-behavior therapy for obsessive-compulsive disorder. Cognitive Behavior Therapy in Korea 2007;7:17-40.

29. Yoon HY, Jeong SH, Shin MS, Kim JS, Kim MS, Kwon JS. Group cognitive behavioral treatment of obsessive-compulsive disorder. Korean J Psychopathol 2000;9:142-156.

30. Foa EB, Kozak MJ. Mastery of Obsessive-Compulsive Disorder: a Cognitive-Behavioral Approach Therapist Guide. New York: Oxford University Press; 2004.

31. Salkovskis PM, Kirk J. Obsessive-Compulsive Disorder. In: Clark DM, Fairburn CG, Editors. Science and Practice of Cognitive Behaviour Therapy. New York: Oxford University Press, 1997, p. 179-208.

32. Seol SH, Kwon JS, Shin MS. Korean self-report version of the YaleBrown Obsessive-Compulsive Scale: factor structure, reliability, and validity. Psychiatry Investig 2013;10:17-25.

33. Beck AT, Steer RA, Carbin MG. Psychometric properties of the Beck Depression Inventory: twenty-five years of evaluation. Clin Psychol Rev 1988;8:77-100.

34. Beck A, Steer R. Beck Anxiety Inventory Manual. San Antonio, TX: The Psychological Corporation; 1993.

35. Mundt JC, Marks IM, Shear MK, Greist JH. The Work and Social Adjustment Scale: a simple measure of impairment in functioning. Br J Psychiatry 2002;180:461-464.

36. Olley A, Malhi G, Sachdev P. Memory and executive functioning in obsessive-compulsive disorder: a selective review. J Affect Disord 2007; 104:15-23.

37. Reitan RM, Wolfson D. The Halstead-Reitan Neuropsychological Test Battery: Theory and Clinical Interpretation. Tucson: Neuropsychology Press; 1985.

38. Kim JK, Kang YW. Korean-California Verbal Learning Test. Seoul: The Psychological Company; 1997.

39. Kim HK. Rey-Kim Memory Test. Daegu: Korea Neuropsychology Press; 1999.

40. Kim HK. Kims Frontal-Executive Neuropsychological Test. Daegu: Korea Neuropsychology Press; 2001.

41. Freedman M, Black S, Ebert P, Binns M. Orbitofrontal function, object alternation and perseveration. Cereb Cortex 1998;8:18-27.

42. Heaton RK. Wisconsin Card Sorting Test: Computer Version 2. Odessa: Psychological Assessment Resources; 1993.

43. Yum TH, Park YS, Oh KJ, Kim JG, Lee YH. The manual of KoreanWechsler Adult Intelligence Scale. Seoul: Korean Guidance Press; 1992.

44. Fisher PL, Wells A. How effective are cognitive and behavioral treatments for obsessive-compulsive disorder? A clinical significance analysis. Behav Res Ther 2005;43:1543-1558.

45. Farris SG, McLean CP, Van Meter PE, Simpson HB, Foa EB. Treatment response, symptom remission, and wellness in obsessive-compulsive disorder. J Clin Psychiatry 2013;74:685-690.

46. Marks IM, Cavanagh K, Gega L. CP for Obsessive-Compulsive Disorder and Post-Traumatic Stress Disorder. In: Marks IM, Cavanagh K, Gega L, Editors. Hands-on Help: Computer-Aided Psychotherapy. Hove and New York: Psychology Press, 2007, p. 61-72.

47. Steketee G, Barlow DH. Obsessive-Compulsive Disorder. In: Barlow DH, Editor. Anxiety and its Disorders: the Nature and Treatment of Anxiety and Panic. New York: The Guilford Press, 2004, p. 516-550. 
48. Leyfer OT, Ruberg JL, Woodruff-Borden J. Examination of the utility of the Beck Anxiety Inventory and its factors as a screener for anxiety disorders. J Anxiety Disord 2006;20:444-458.

49. Novara C, Pastore M, Ghisi M, Sica C, Sanavio E, McKay D. Longitudinal aspects of obsessive compulsive cognitions in a non-clinical sample: a five-year follow-up study. J Behav Ther Exp Psychiatry 2011;42:317324.

50. Han E, Cho Y, Park S, Kim H, Kim S. Factor structure of the Korean version of the Beck Anxiety Inventory: an application of confirmatory factor analysis in psychiatric patients. Kor J Clin Psychol 2003;22:261-270.

51. Lenhard F, Vigerland S, Andersson E, Rück C, Mataix-Cols D, Thulin U, et al. Internet-delivered cognitivie behavior therapy for adolescents with obsessive-copulsive disorder: an open trial. PLoS One 2014;9:e100773.

52. Simpson HB, Maher MJ, Wang Y, Bao Y, Foa EB, Franklin M. Patient adherence predicts outcome from cognitive behavioral therapy in obsessive-compulsive disorder. J Consult Clin Psychol 2011;79:247-252.
53. Olatunji BO, Davis ML, Powers MB, Smits JA. Cognitive-behavioral therapy for obsessive-compulsive disorder: a meta-analysis of treatment outcome and moderators. J Psychiatr Res 2013;47:33-41.

54. Huppert JD, Franklin ME. Cognitive behavioral therapy for obsessivecompulsive disorder: an update. Curr Psychiatry Rep 2005;7:268-273.

55. Mewton L, Sachdev PS, Andrews G. A naturalistic study of the acceptability and effectiveness of internet-delivered cognitive behavioural therapy for psychiatric disorders in older australians. PLoS One 2013;8: e71825.

56. Moritz S, Kloss M, Jacobsen D, Fricke S, Carrie C, Brassen S, et al. Neurocognitive impairment does not predict treatment outcome in obsessive-compulsive disorder. Behav Res Ther 2005;43:811-819.

57. D’Alcante CC, Diniz JB, Fossaluza V, Batistuzzo MC, Lopes AC, Shavitt RG, et al. Neuropsychological predictors of response to randomized treatment in obsessive-compulsive disorder. Prog Neuropsychopharmacol Biol Psychiatry 2012;39:310-317. 